\title{
The dynamics and interactions of Scs proteins from Proteus mirabilis
}

\author{
Andrew Whitten ${ }^{1}$, Furlong Emily², Choudhury Hassanul ${ }^{2}$, Kurth Fabian², Duff Anthony ${ }^{1}$, Martin Jennifer ${ }^{2}$ \\ ${ }^{I}$ Australian Nuclear Science and Technology Organisation, Lucas Heights, Australia; \\ ${ }^{2}$ Institute for Molecular Bioscience, University of Queensland, Australia \\ awh@ansto.gov.au
}

Correct formation of disulfide bonds is critical to the folding of a wide variety of proteins. Bacterial virulence factors are one class of proteins containing disulfide bonds, thus, an approach to disarm virulent bacterial might involve shutting down the machinery involved in the formation of disulfide bonds. The suppressor of copper sensitivity (Scs) proteins form part of the disulfide bond forming machinery in bacteria, and it is hoped that determining the structure of molecules such as this may lead to the development of new classes of antibiotics. There are four Scs proteins (ScsA, B, C and D) present in numerous Gram-negative bacteria, and few have been structurally characterised. In this work we show that the ScsC protein from Proteus mirabilis is trimeric and flexible, where the high level of flexibility is afforded by a glutamine rich motif. We also show that the protein interacts with ScsB and that this interaction rigidifies the $\mathrm{ScsC}$ protein.

Keywords: Small-angle scattering; protein complex; protein dynamics 